C. Grosche F. Steiner

\title{
Handbook \\ of Feynman Path Integrals
}




\section{Table of Contents}

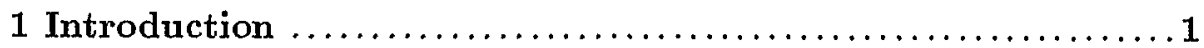

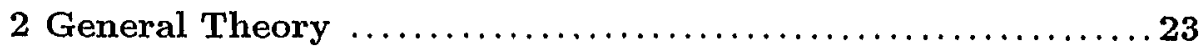

2.1 The Feynman Kernel and the Green Function ...............23

2.2 The Path Integral in Cartesian Coordinates .................. 31

2.3 Gaussian Path Integrals and Zeta Function Regularization .......37

2.4 Evaluation of Path Integrals by Fourier Series ................39

2.5 Path Integration Over Coherent States .................... 44

2.6 Fermionic Path Integrals .............................55

2.7 The Path Integral in Spherical Coordinates .................63

2.8 The Path Integral in General Coordinates ................. 67

2.9 Transformation Techniques $\ldots \ldots \ldots \ldots \ldots \ldots \ldots \ldots \ldots \ldots \ldots$

2.10 Exact Path Integral Treatment of the Hydrogen Atom ......... 87

2.11 The Path Integral in Parabolic Coordinates .................88

3 Basic Path Integrals $\ldots \ldots \ldots \ldots \ldots \ldots \ldots \ldots \ldots \ldots \ldots \ldots \ldots . \ldots 9$

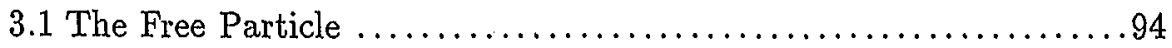

3.2 The Quadratic Lagrangian $\ldots \ldots \ldots \ldots \ldots \ldots \ldots \ldots \ldots \ldots \ldots \ldots$

3.3 The Radial Harmonic Oscillator ....................... 99

3.4 Path Integration Over Group Manifolds ...................102

4 Perturbation Theory $\ldots \ldots \ldots \ldots \ldots \ldots \ldots \ldots \ldots \ldots \ldots \ldots \ldots, 123$

4.1 Path Integration and Perturbation Theory ................. 124

4.2 Summation of the Perturbation Series for $\delta$ - and $\delta^{\prime}$-Potentials $\ldots . .127$

4.3 Partition Functions and Effective Potentials ............... 132

4.4 Semiclassical Expansion About the Harmonic Approximation .... 134 


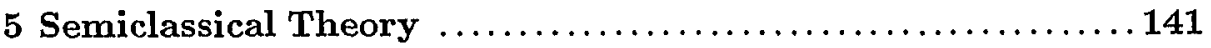

5.1 Semiclassical Theory and Quantum Chaos ................. 141

5.2 Semiclassical Expansion of the Feynman Path Integral ......... 143

5.3 Semiclassical Expansion of the Green Function ................ 149

5.4 The Gutzwiller Trace Formula .......................... 151

6 Table of Path Integrals ............................. 155

6.1 General Formulæ ................................. 162

6.2 The General Quadratic Lagrangian ...................... 173

6.3 Discontinuous Potentials .......................... 217

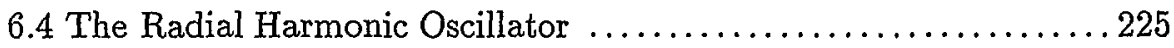

6.5 The Pöschl-Teller Potential .......................... 240

6.6 The Modified Pöschl-Teller Potential ....................244

6.7 Motion on Group Spaces and Homogeneous Spaces ........... 258

6.8 Coulomb Potentials ............................... 277

6.9 Magnetic Monopole and Anyon Systems ..................295

6.10 Motion in Hyperbolic Geometry .......................304

6.11 Explicit Time-Dependent Problems ....................322

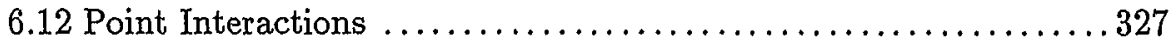

6.13 Boundary Value Problems ........................... 347

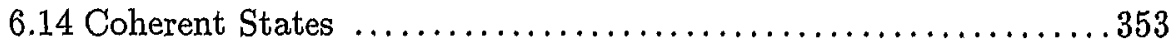

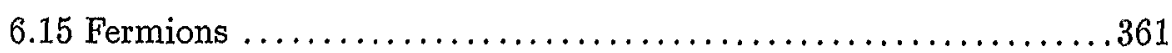

6.16 Supersymmetric Quantum Mechanics ...................363

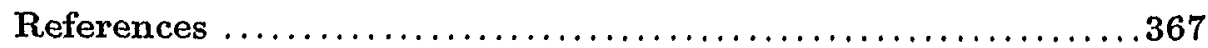

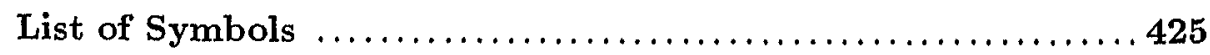

Subject Index $\ldots \ldots \ldots \ldots \ldots \ldots \ldots \ldots \ldots \ldots \ldots \ldots \ldots \ldots \ldots, \ldots 29$

Author Index $\ldots \ldots \ldots \ldots \ldots \ldots \ldots \ldots \ldots \ldots \ldots \ldots \ldots \ldots \ldots, 439$ 